\title{
Fetal Exposure to MR Imaging: Long-Term Neurodevelopmental Outcome
}

\author{
(D)E. Zvi, (D)A. Shemer, (D). toussia-Cohen, (DD. Zvi, (D). Bashan, (D). Hirschfeld-dicker, (DN. Oselka, (D) M.-M. Amitai, (D) O. Ezra,
}

(D) O. Bar-Yosef, and (DE. Katorza

\begin{abstract}
BACKGROUND AND PURPOSE: Very few studies have investigated long-term neurodevelopment of children exposed to MR imaging antenatally. Thus, the purpose of our study was to evaluate long-term neurodevelopmental outcomes of children exposed to MR imaging during pregnancy.
\end{abstract}

MATERIALS AND METHODS: We conducted a historical prospective cohort study in a single tertiary medical center. Women exposed to 1.5T noncontrast MR imaging for maternal or fetal indications were matched to unexposed controls. Long-term neurodevelopmental outcomes were evaluated of their children, 2.5 to 6 years of age, according to the Vineland-II Adaptive Behavior Scale. The Vineland-II Adaptive Behavior Scale assesses communication, daily living skills, socialization, and motor skills. A composite score summarizes these 4 domains.

RESULTS: A total of 131 exposed women matched our inclusion criteria and were included in the study group, and 771 unexposed women, in the control group. No difference was identified in the Vineland-II Adaptive Behavior Scale composite score between the children of the study and control groups (mean, 110.79 versus 108.18; $P=.098$ ). Differences were also not observed between the children of the 2 groups in 3 of the 4 questionnaire domains: communication (108.84 versus 109.10; $P=.888$ ), daily living skills (109.51 versus 108.28; $P=.437$ ), and motor skills (105.09 versus 104.42; $P=.642$ ). However, the socialization score was favorable for the study group (112.98 versus $106.47 ; P<.001)$

CONCLUSIONS: Exposure to 1.5T noncontrast MR imaging during pregnancy had no harmful effects on long-term neurodevelopmental outcomes. This study contributes to understanding the safety of MR imaging during pregnancy.

ABBREVIATION: VABS $=$ Vineland-II Adaptive Behavior Scale

M $\mathrm{R}$ imaging has become commonly used when higher resolution imaging is needed for fetuses and pregnant women. The consensus is to avoid ionizing radiation whenever possible due to adverse effects on fetal development. ${ }^{1,2}$ Therefore, clinicians prefer to use nonionizing imaging such as sonography and MR imaging during pregnancy. ${ }^{3}$

Received May 25, 2020; accepted after revision July 14.

From the Antenatal Diagnostic Unit (E.Z., S.t.-C., O.E., E.K.), Department of Obstetrics and Gynecology, and Department of Diagnostic Radiology (M.-M.A.) Chaim Sheba Medical Center, Tel-Hashomer, Israel; Sackler School of Medicine (E.Z., A.S., S.t.-C., D.Z., Y.B., L.H.-d., N.O., M.-M.A., O.E., O.B.-Y., E.K.), Tel Aviv University, Tel Aviv, Israel; and Pediatric Neurology Unit (O.B.-Y.), Sheba Medical Center, Ramat-Gan, Israel.

Paper previously presented as a poster at: Sackler Faculty of Medicine 13th Medical Research Fair, April 16, 2019; Tel Aviv, Israel.

Please address correspondence to Elad Zvi, MD, Chaim Sheba Medical Center, Tel-Hashomer 52621, Ramat Gan, Israel; e-mail: elad.zvi@gmail.com

Indicates article with supplemental on-line tables.

http://dx.doi.org/10.3174/ajnr.A6771
Currently, MR imaging is used for fetal and maternal indications in pregnant women with selected conditions. ${ }^{4,5}$ Animal studies have shown associations of some adverse effects with long-term exposure to MR imaging, including hearing impairment, skeletal malformations, and fetal weight abnormalities. ${ }^{6-8}$ Human studies did not show similar results but concluded that more research needs to be conducted. ${ }^{9-11}$

The American College of Radiology practice guidelines state that there is no conclusive evidence of harmful effects on a developing fetus exposed to $1.5 \mathrm{~T}$ MR imaging. ${ }^{12}$ However, concerns about MR imaging safety during pregnancy, especially during organogenesis, have been raised due to loud noise, heating caused by absorption of radio waves, and exposure to high-power electromagnetic fields. ${ }^{13-16}$ Two recent studies with large cohorts helped shed light on the subject. One retrospective study compared hearing screening results of 751 neonates exposed to MR imaging in utero with those of 10,042 unexposed neonates. The respective rates of hearing impairment or deafness at birth were 
$0 \%$ and $0.34 \%$. Long-term outcomes were not assessed. The median gestational age at MR imaging was 37 weeks, with the earliest at 16 weeks. ${ }^{17}$ The second study compared 1737 fetuses exposed to MR imaging during the first trimester versus $1,418,451$ unexposed fetuses. A follow-up at 4 years of age was performed using diagnosis billing codes. Five outcomes were evaluated: 1) stillbirth after 20 weeks' gestational age, 2) congenital anomaly, 3) neoplasm, 4) vision loss, and 5) hearing loss. The risk for these outcomes was not significantly higher in the exposed compared with the unexposed group. ${ }^{18}$ To the best of our knowledge, studies of long-term neurodevelopmental assessments are scarce. Thus, the objective of our study was to examine the long-term neurodevelopmental outcomes of fetuses exposed to MR imaging during pregnancy.

\section{MATERIALS AND METHODS}

This is a historical prospective cohort study, conducted in a single tertiary medical center (Chaim Sheba Medical Center) between 2011 and 2017. Demographic and clinical data were collected from the electronic records of each patient. The study included all women who gave birth at our medical center and underwent a noncontrast 1.5T MR imaging during pregnancy due to fetal or maternal indications. Maternal indications for MR imaging included suspected appendicitis, cholecystitis, multiple sclerosis, back pain, severe headaches, and trauma. Fetal indications for MR imaging included suspected mild ventricular dilation or asymmetry, ${ }^{19}$ microcephaly, or macrocephaly and a previous pregnancy with CNS malformations. All the fetuses included in the study had normal MR imaging findings and were without evidence of chromosomal abnormalities or cytomegalovirus infection during pregnancy. No contrast materials were used during the MR imaging. Women with substance abuse, including tobacco and alcohol, were excluded from the study. We created a control group that consisted of women who gave birth at the same medical center on the same days. All the women in the control group met the inclusion and exclusion criteria of the study, apart from exposure to MR imaging during pregnancy.

For the neurodevelopmental assessment, we selected women from the control group and matched them according to date of birth and mode of delivery at the ratio of 1:1 with the study group. Four neurodevelopmental long-term aspects were assessed using the Vineland-II Adaptive Behavior Scale (VABS): communication, daily living skills, socialization, and motor skills. These provide a composite score that summarizes the individual's performance across all 4 domains. ${ }^{20,21}$ Telephone interviews were conducted between February 2017 and July 2017. All the children were between 2.5 and 6 years of age at the time of the interviews. The VABS is standardized by age. The mean score for all ages is $100 \pm 15$. Scores of $<70$ are considered in the abnormal range.

Categoric variables were described using frequencies and percentages. Continuous variables were evaluated for normal distribution using histograms and quartile-quartile plots. Normally distributed continuous variables were described as means and SDs, and skewed variables were expressed as medians and interquartile ranges. The ANOVA, independentsamples $t$ test, Kruskal-Wallis test, and the Mann-Whitney test were used, as appropriate, to compare continuous variables among categories within the exposed group. Categoric variables were compared using the $\chi^{2}$ test or Fisher exact test. Study and control-matched women were compared using the paired-samples $t$ test, Wilcoxon signed rank test, McNemar test, generalized estimating equations, or conditional logistic regression, as appropriate.

The sample size was calculated by using WINPEPI software (http://www.brixtonhealth.com/pepi4windows.html). Significance level and power were set to $5 \%$ and $80 \%$, respectively. To identify a 5-point difference in the VABS composite score, 73 matched pairs of patients were needed.

All the statistical tests were 2 -tailed. $P<.05$ was considered statistically significant. All the statistical analyses were performed using SPSS Statistics software, Version 24 (IBM).

The study was approved by the institutional review board (No. 1902-15-SMC). The authors obtained both informed consent and ethics committee approval for accessing data from patient records and conducting a telephone questionnaire.

\section{RESULTS}

\section{Demographic and Clinical Characteristics of the Study Population}

One hundred thirty-one pregnant women who were exposed to MR imaging during pregnancy, between 2011 and 2015 met the inclusion criteria of the study. The excluded cases were mainly due to fetal anomalies or chromosomal aberrations, twin pregnancy, cytomegalovirus infection during pregnancy, and delivery in a different medical center. Of 131 deliveries, 58 (44.3\%) were girls; the mean gestational age at birth was 39 weeks. Eight women were exposed to MR imaging during the first trimester; 28, during the second; and 95, during the third. During pregnancy, 114 women were exposed once to MR imaging scans, and 17 were exposed $\geq 2$ times. Seventy-two MR imaging scans were for fetal indications ( $68 \mathrm{CNS}$ and 4 body), and 62 were for maternal indications (20 CNS and 42 body) as shown in On-line Table 1.

\section{Comparison between the Study and Control Groups}

During the same study period, 771 women met our criteria for the control group. Of them, 392 fetuses were girls (50.8\%). The mean gestational age at birth was 39 weeks. Compared with the control group, for the study group, parity was higher (1.68 versus $1.14)$, as was the prevalence of pregestational diabetes mellitus (6.1\% versus $1.3 \%$ ), the use of anticoagulation (8.4\% versus $3.9 \%$ ) and antihypertensive medications during pregnancy (6.9\% versus $1.2 \%$ ), and cesarean delivery (37\% versus $22.8 \%$ ). In contrast, thrombocytopenia was more prevalent in the control than in the study group (16\% versus 3.8\%). Statistically significant differences were not found between the groups in maternal age during delivery or in the mode of conception, as shown in On-line Table 2.

The neonatal characteristics and outcomes for the study and control groups are presented in On-line Table 3. Compared with the study group, for the control group, the mean gestational age at delivery was higher (39.01 versus 38.41 weeks, $P<.001$ ) and the birth weight was higher (3216.6 versus $3069.95 \mathrm{~g}, P=.002$ ). The weight percentiles did not differ significantly between the 
groups. The mean APGAR scores after 5 minutes were higher in the control than the study group (9.96 versus 9.86, $P=.04$ ). APGAR 1-minute scores and hearing test results after birth did not differ between the groups.

\section{Neurodevelopmental Outcome}

One hundred ninety-two women responded to the VABS in a 1:1 ratio of exposed and unexposed cases, matched according to the birth date and mode of delivery (96 in each group). No statistically significant difference was found in the VABS composite score between the children of the study and control groups (110.79 versus $108.18, P=.098$ ). None of the children (exposed or nonexposed) had abnormal VABS composite scores. However, analyzing each domain separately (communication, daily living skills, socialization, and motor skills), a statistically significant difference was noticed between the groups only in the socialization score in favor of the study group $(112.98 \pm 13.28$ versus $106.47 \pm 11.08$, $P<$.001) (On-line Table 4).

In an analysis that stratified by the trimester of MR imaging exposure (On-line Table 5), the mean motor skills score of the VABS was significantly lower among those exposed in the first compared with the second and third trimesters $(94.5 \pm 10.71,106.28 \pm$ 11.03 , and $105.91 \pm 10.31$, respectively, $P=.037)$. Notably, despite the statistically significant difference, all the scores were within the normal range. No other differences in fetal outcome (birth weight, APGAR score, hearing screening) were found according to the trimester of exposure, the number of MR imaging exposures during the same pregnancy, and the MR imaging protocols.

\section{DISCUSSION}

Our study evaluated long-term neurodevelopmental outcomes of children exposed to MR imaging during pregnancy, according to VABS, as assessed by telephone interviews.

We showed no difference in the adaptive behavior composite score between the exposed and the unexposed groups.

However, the socialization score of the study group was significantly higher than that of the control group (112.98 versus 106.47; $P<.001)$. This finding probably does not have any clinical significance because all the scores are within the normal range. Our results suggest that exposure to MR imaging does not have a harmful long-term effect on the child's neurodevelopment between 2.5 and 6 years of age. This result is in agreement with those in smaller studies that evaluated long-term effects of fetal MR imaging exposure. ${ }^{22,23}$

Recommendations that have been published for MR imaging safety during pregnancy do not contraindicate the use of this technique according to the trimester of pregnancy. ${ }^{3,14,24}$ Other studies have suggested that MR imaging is safe during all trimesters of pregnancy in regard to neonatal normal hearing and vision and the risks for neoplasm, stillbirth, and congenital anomalies. ${ }^{17,18}$ No other study evaluated long-term neurodevelopment of MR imaging-exposed fetuses or compared the MR imaging exposure effects in different trimesters and protocols. The lack of long-term adverse effects demonstrated in our study corroborates previous reports and supports the use of MR imaging in prenatal imaging.

We found that children who were exposed to MR imaging in the first trimester had lower scores on the motor skills domain of the VABS than those exposed in the second and third trimesters. This is probably without any clinical manifestation because all the scores were within the normal range and all the neonates were delivered at term with normal APGAR scores. However, only 8 fetuses were exposed in the first trimester.

None of the outcomes examined differed between children whose mothers underwent a CNS protocol MR imaging during pregnancy and children whose mothers underwent other MR imaging protocols. Notably, in mothers with CNS protocols, the distance of the fetus from the center of the MR imaging is farther than with other protocols; therefore, the exposure is lower. ${ }^{25}$ A similar result was demonstrated when comparing the fetal CNS protocol, in which the MR imaging is focused on the fetal brain, with the rest of the protocols. Thus, according to these findings, the fetus is not affected by MR imaging, regardless of the protocol used.

The proportion of cesarean deliveries was higher in the study than in the control group. A possible explanation may relate to close monitoring of most of the pregnancies of women who underwent MR imaging due to the particular circumstances involved. No statistically significant differences were noticed between the groups regarding birth weight, weight percentile, and 5-minute APGAR scores, concurring with previous reports. ${ }^{26}$ Regarding birth weight and gestational age at delivery, we showed similar results to those in a previous study. ${ }^{27}$

The strengths of this study include the long-term evaluation of motor skills, socialization, daily living skills, and communication obtained by the VABS. In addition, to avoid any confounders that could affect the results, we set strict inclusion and exclusion criteria that were achieved by reviewing all the medical files. All information regarding prenatal evaluation before the MR imaging exposure was collected, together with potential teratogens such as medication, alcohol use, and maternal morbidity. Furthermore, all the MR imaging protocols are known and were grouped by anatomic region of the mother and fetus. To further reduce any confounders, we matched 96 women in the study and control groups by day and mode of delivery.

Our study has a number of limitations. First, only a small number of women were exposed to MR imaging during the first trimester, thus limiting the power of this study to assess the risk of MR imaging exposure during this trimester. Second, the main indication of MR imaging in this study was for the fetal CNS (35.3\%), which may cause a selection bias.

\section{CONCLUSIONS}

Exposure to $1.5 \mathrm{~T}$ noncontrast MR imaging during pregnancy had no harmful effects on long-term neurodevelopmental outcomes, regardless of gestational age, the number of MR imaging scans during pregnancy, and the use of various MR imaging protocols. This study contributes to the understanding of the safety of MR imaging during pregnancy and may benefit prenatal counseling.

\section{REFERENCES}

1. Fazel R, Krumholz HM, Wang Y, et al. Exposure to low-dose ionizing radiation from medical imaging procedures. $N \mathrm{Engl} \mathrm{J} \mathrm{Med}$ 2009;361:849-57 CrossRef Medline 
2. Chen MM, Coakley FV, Kaimal A, et al. Guidelines for computed tomography and magnetic resonance imaging use during pregnancy and lactation. Obstet Gynecol 2008;112:333-40 CrossRef Medline

3. ACOG Committee on Obstetric Practice. ACOG Committee Opinion: Number 299, September 2004 (replaces No. 158, September 1995)— guidelines for diagnostic imaging during pregnancy. Obstet Gynecol 2004;104:647-51 CrossRef Medline

4. Rapp EJ, Naim F, Kadivar K, et al. Integrating MR imaging into the clinical workup of pregnant patients suspected of having appendicitis is associated with a lower negative laparotomy rate: singleinstitution study. Radiology 2013;267:137-44 CrossRef Medline

5. Spalluto LB, Woodfield CA, DeBenedectis CM, et al. MR imaging evaluation of abdominal pain during pregnancy: appendicitis and other nonobstetric causes. Radiographics 2012;32:317-34 CrossRef Medline

6. Mevissen M, Buntenkötter S, Löscher W. Effects of static and timevarying $(50-\mathrm{Hz})$ magnetic fields on reproduction and fetal development in rats. Teratology 1994;50:229-37 CrossRef Medline

7. Yip YP, Capriotti C, Talagala SL, et al. Effects of MR exposure at $\mathbf{1 . 5}$ T on early embryonic development of the chick. J Magn Reson Imaging 1994;4:742-48 CrossRef Medline

8. Gerhardt KJ, Pierson LL, Huang X, et al. Effects of intense noise exposure on fetal sheep auditory brain stem response and inner ear histology. Ear Hear 1999;20:21-32 CrossRef Medline

9. Rocha EB, Frasson de Azevedo M, Ximenes Filho JA. Study of the hearing in children born from pregnant women exposed to occupational noise: assessment by distortion product otoacoustic emissions. Braz J Otorhinolaryngol 2007;73:359-69 CrossRef Medline

10. Reeves MJ, Brandreth M, Whitby EH, et al. Neonatal cochlear function: measurement after exposure to acoustic noise during in utero MR imaging. Radiology 2010;257:802-09 CrossRef Medline

11. Choi JS, Ahn HK, Han JY, et al. A case series of $\mathbf{1 5}$ women inadvertently exposed to magnetic resonance imaging in the first trimester of pregnancy. J Obstet Gynaecol 2015;35:871-72 CrossRef Medline

12. American College of Radiology. ACR-SPR Practice Guideline for the Safe and Optimal Performance of Fetal Magnetic Resonance Imaging (MRI). American College of Radiology; 2015. https://www. acr.org/-/media/ACR/Files/Practice-Parameters/mr-fetal.pdf. Accessed November 13, 2016

13. Patenaude Y, Pugash D, Lim K, et al; Society of Obstetricians and Gynaecologists of Canada, The use of magnetic resonance imaging in the obstetric patient. J Obstet Gynaecol Can 2014;36:349-63 CrossRef Medline
14. Kanal E, Barkovich AJ, Bell C, et al; ACR Blue Ribbon Panel on MR Safety. ACR guidance document for safe MR practices: 2007. AJR Am J Roentgenol 2007;188:1447-74 CrossRef Medline

15. Hartwig V, Giovannetti G, Vanello N, et al. Biological effects and safety in magnetic resonance imaging: a review. Int $J$ Environ Res Public Health 2009;6:1778-98 CrossRef Medline

16. Kikuchi S, Saito K, Takahashi M, et al. Temperature elevation in the fetus from electromagnetic exposure during magnetic resonance imaging. Phys Med Biol 2010;55:2411-26 CrossRef Medline

17. Strizek B, Jani JC, Mucyo E, et al. Safety of MR imaging at $1.5 \mathrm{~T}$ in fetuses: a retrospective case-control study of birth weights and the effects of acoustic noise. Radiology 2015;275:530-37 CrossRef Medline

18. Ray JG, Vermeulen MJ, Bharatha A, et al. Association between MRI exposure during pregnancy and fetal and childhood outcomes. JAMA 2016;316:952-61 CrossRef Medline

19. Barzilay E, Bar-Yosef O, Dorembus S, et al. Fetal brain anomalies associated with ventriculomegaly or asymmetry: an MRI-based study. AJNR Am J Neuroradiol 2017;38:371-75 CrossRef Medline

20. Limperopoulos C, Majnemer A, Steinbach CL, et al. Equivalence reliability of the Vineland Adaptive Behavior Scale between in-person and telephone administration. Phys Occup Ther Pediatr 2006;26:11527 CrossRef Medline

21. Sparrow SS, Balla DA, Cicchetti DV. PsycTESTS dataset. In: Sparrow SS, Balla DA, Cicchetti DV. Vineland Adaptive Behavior Scales. 2nd ed. American Psychological Association; 2005

22. Clements H, Duncan KR, Fielding K, et al. Infants exposed to MRI in utero have a normal paediatric assessment at 9 months of age. Br J Radiol 2000;73:190-94 CrossRef Medline

23. Kok RD, de Vries MM, Heerschap A, et al. Absence of harmful effects of magnetic resonance exposure at $1.5 \mathrm{~T}$ in utero during the third trimester of pregnancy: a follow-up study. Magn Reson Imaging 2004;22:851-54 CrossRef Medline

24. Jaffe TA, Miller CM, Merkle EM. Practice patterns in imaging of the pregnant patient with abdominal pain: a survey of academic centers. AJR Am J Roentgenol 2007;189:1128-34 CrossRef Medline

25. Pediaditis M, Leitgeb N, Cech R. RF-EMF exposure of fetus and mother during magnetic resonance imaging. Phys Med Biol 2008;53:7187-95 CrossRef Medline

26. Dollberg S, Haklai Z, Mimouni FB, et al. Birth weight standards in the live-born population in Israel. Isr Med Assoc J 2005;7:311-14 Medline

27. Myers C, Duncan KR, Gowland PA, et al. Failure to detect intrauterine growth restriction following in utero exposure to MRI. $\mathrm{Br} J$ Radiol 1998;71:549-51 CrossRef Medline 